\title{
Celastrol Niosome Hydrogel Has Anti-Inflammatory Effect on Skin Keratinocytes and Circulation without Systemic Drug Exposure in Psoriasis Mice
}

\author{
Fen Qiu ${ }^{1,2, *}$ \\ Long $\mathrm{Xi}^{2, *}$ \\ Shengshuang Chen ${ }^{3}$ \\ Yonghua Zhao ${ }^{2,4}$ \\ Zhenping Wang ${ }^{5}$ \\ Ying Zheng $\mathbb{D}^{2}$
}

'State Key Laboratory of Southwestern Chinese Medicine Resources, Chengdu University of Traditional Chinese Medicine, Chengdu, 61 II37, People's Republic of China; ${ }^{2}$ State Key Laboratory of Quality Research in Chinese Medicine, Institute of Chinese Medical Sciences, University of Macau, Macau, People's Republic of China; ${ }^{3}$ Macau Institute for Applied Research in Medicine and Health, State Key Laboratory of Quality Research in Chinese Medicine, Macau University of Science and Technology, Macau SAR, People's Republic of China; ${ }^{4}$ GuangdongHong Kong-Macau Joint Laboratory on Chinese Medicine and Immune Disease Research, University of Macau, Macau, People's Republic of China; ${ }^{5}$ Department of Dermatology, School of Medicine, University of California, San Diego, La Jolla, CA, USA

*These authors contributed equally to this work

Correspondence: Ying Zheng State Key Laboratory of Quality Research in Chinese Medicine, Institute of Chinese Medical Sciences, University of Macau

Tel +85388224687

Fax +85328841358

Email yzheng@umac.mo
Purpose: Psoriasis is an inflammatory skin disease, where keratinocytes play pivotal roles in its pathogenesis. We prepared Celastrol Noisome hydrogel (Cel Nio gel) for the treatment of psoriasis and aimed to study its target site as well as the mechanism.

Methods: Cel Nio was fabricated with thin-film hydration and sonication, then topically administered to imiquimod (IMQ)-induced psoriasis mice. The concentrations of Cel in the skin, blood and lymphatic system were determined using LC-MS. The anti-psoriasis effect of Cel Nio gel was studied, and the levels of inflammatory cytokines in blood were evaluated by flow cytometry. For the in vitro study, the uptake of Nio by $\mathrm{HaCaT}$ cells was quantified with flow cytometry, and the anti-inflammatory effect of $\mathrm{Cel}$ on $\mathrm{HaCaT}$ cells was detected with qPCR. The expressions of inflammatory factors and Ki-67 in skin were observed by immunofluorescence.

Results: Cel Nio possessed a particle size of $133 \mathrm{~nm}$ with encapsulation efficacy (EE\%) of $83.2 \%$. After topical administration of Cel Nio gel to mice, Cel was mainly accumulated in the skin instead of exposure in blood or lymphatic system, while the levels of inflammatory factors in blood had a significant decline. In addition, the preparation of Nio enhanced the uptake by $\mathrm{HaCaT}$ cells, and Cel obviously reduced the mRNA levels of inflammatory cytokines in HaCaT cells. Moreover, Cel Nio gel significantly decreased the expression of inflammatory cytokines and $\mathrm{Ki}-67$ in the skin.

Conclusion: Cel Nio gel achieved the anti-psoriatic effect by inhibiting the inflammation and hyperproliferation of keratinocytes in the skin and further suppressing the systemic inflammation, thus could be a novel topical drug delivery system to treat psoriasis with topical and systemic effects.

Keywords: psoriasis, celastrol niosome, topical delivery, keratinocytes, pharmacokinetics

\section{Introduction}

Psoriasis is a chronic skin disease that affects more than 100 million individuals around the world. ${ }^{1}$ The typical symptoms of psoriasis are erythema, itchiness, and scaliness, which result in physical discomfort and psychological burden. ${ }^{2}$ Psoriasis is a disorder that involves both the innate and adaptive immune systems, where keratinocytes, dendritic cells (DCs), and T cells play vital roles. ${ }^{3,4}$ Psoriatic keratinocytes initiate the innate immune system in the early stage of psoriasis, which involve the adaptive response and reserve inflammatory mediators in the development of psoriasis. ${ }^{5}$ In the early stages of psoriasis, keratinocytes are activated by the antimicrobial peptide LL37 to produce interferon- $\beta$ (IFN- $\beta$ ) and other cytokines, which promote the maturation of DCs. ${ }^{6}$ Subsequently, mature DCs release 


\section{Graphical Abstract}

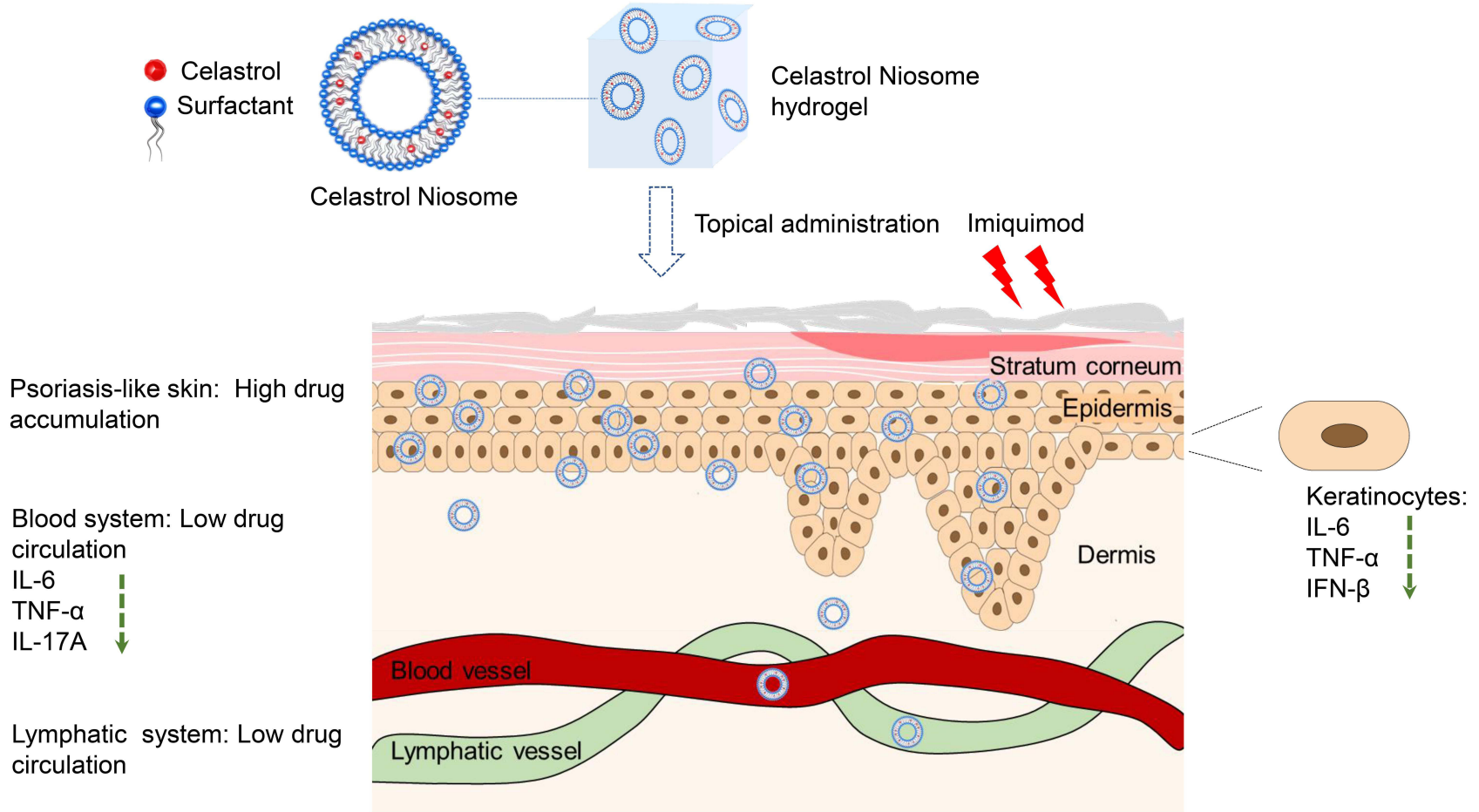

Schematic illustration of accumulation and bioactivity of Celastrol Niosome hydrogel (Cel Nio gel) in imiquimod-induced psoriasis mice. Cel Nio gel was topically administrated on the imiquimod-induced psoriasis mice model, which was mainly accumulated in the skin without obvious exposure to blood and lymphatic system. Cel Nio gel inhibited the inflammation of keratinocytes in the skin and further suppressed the systemic inflammatory response in the system.

interleukin -23 (IL-23), tumor necrosis factor- $\alpha$ (TNF- $\alpha$ ) and other cytokines, which strongly activate the differentiation of naive $\mathrm{T}$ cells into $\mathrm{T}$ helper cells to release interferon- $\gamma$ (IFN- $\gamma$ ), interleukin-17 (IL-17), and so on. ${ }^{7}$ These cytokines further induce the major histopathological symptoms of psoriasis, including hyperplasia of keratinocytes and inflammatory cell infiltration. ${ }^{8}$

The treatments for psoriasis mainly include topical therapies, phototherapy, oral systemic medications, and biological agents. ${ }^{9}$ In the last few years, biological agents targeted to key cytokines that targeted to the IL-23/IL-17 axis have been the most efficacious therapeutics for psoriasis vulgaris, with the drawback of much higher cost than other antipsoriasis treatments. ${ }^{10}$ Despite recent developments in the systemic and biological treatment of psoriasis, topical therapies represent the main treatment for about $80 \%$ of the patients with mild-to-moderate psoriasis. ${ }^{11,12}$ Topical administration has the advantages of reducing the side effects and increasing the therapeutic effects with a prolonged duration of action in the skin. $^{13}$
Tripterygium wilfordii Hook $\mathrm{f}$. (TwHF) is a traditional Chinese medicine, which has been administered to treat skin diseases for several centuries. ${ }^{14}$ TwHF has been reported to have anti-inflammatory and immunosuppressive effects, which has been applied for the therapy of rheumatoid arthritis and psoriasis vulgaris. ${ }^{15,16}$ Celastrol (Cel), a triterpenoid obtained from TwHF with a molecular weight of $450.6 \mathrm{~g} / \mathrm{mol}$, has anti-inflammatory and anticancer properties, ${ }^{17}$ which can limit the production of Th17-related cytokines and chemokines. ${ }^{18}$ Despite its promising anti-inflammatory and anticancer effects, Cel has also been reported to induce toxicity, as indicated in the weight loss of treated mice. ${ }^{19}$ Besides, the low aqueous solubility $\left(13.25 \pm 0.83 \mu \mathrm{g} / \mathrm{mL}\right.$ at $\left.37{ }^{\circ} \mathrm{C}\right)$ of Cel also restricts its application. ${ }^{20}$

Niosomes (Nios), which are bilayer hydrated vesicular systems consisting of nonionic surfactants and cholesterol, are non-toxic, stable, and low-cost. ${ }^{21}$ Nios are suitable for encapsulating hydrophobic drugs and improving the stability of entrapped active compounds, which have become 
a novel and efficient tool for the delivery of lipophilic drugs. ${ }^{22}$ Besides, drug-loaded Nios have been designed for enhanced management of psoriasis to avoid systemic toxicity. ${ }^{23}$

We have previously prepared Cel Nio gel for topical treatment of IMQ-induced psoriasis. ${ }^{24}$ However, the previous publication did not study the pharmacokinetics of Cel Nio in mice, and the mechanism of the anti-psoriasis effect was not clarified. In the present work, we prepared Cel Nio with thin-film hydration based on our previous study $^{24}$ and further explored the target site and mechanism of Cel Nio gel. The pharmacokinetics of Cel Nio gel was determined by analyzing the accumulation of $\mathrm{Cel}$ in the skin, blood, and lymphatic system using a sensitive LCMS method. Furthermore, the topical and systemic antipsoriasis effects of Cel Nio gel in IMQ-induced psoriatic mice were explored. In addition, the uptake of Nio by $\mathrm{HaCaT}$ cells, the anti-inflammatory activity of $\mathrm{Cel}$ on $\mathrm{HaCaT}$ cells and skin lesions in the IMQ-induced psoriatic mouse model were also explored. The anti-proliferative effect of Cel Nio gel on keratinocytes was also investigated in the IMQ-induced psoriasis mice.

\section{Materials and Methods Materials}

Cholesterol (K1805006), Span 20 (B1806043), and Span 60 (S112961) were obtained from Aladdin company (Shanghai, China). Carbopol powder (0101536985) was purchased from Chineway (Shanghai, China). Cel (19041101) was supplied by Chengdu Pufei De Biotech Co., Ltd. (Chengdu, China). Hair remover spray foam was purchased from Linco Care Ltd. (Carrington, M, USA). IMQ cream (5\%) was obtained from Aldara (Health Care Limited, Loughborough, UK). IMQ powder was acquired from MedChemExpress (Shanghai, China). Protopic $(0.1 \%$, tacrolimus) was supplied by Kangaiduo Drug Store (Guangzhou, China). Absolute ethanol, chloroform, acetonitrile, methanol, and formic acid were from Merck (Darmstadt, Germany). Milli-Q water was produced using a Millipore Direct-Q ultrapure water system (Millipore, Bedford, USA). Goat serum was from Boster Systems, Inc. (AR0009; Pleasanton, CA, USA). Fetal bovine serum (FBS), IFN- $\beta$ (PA5-20390), and ProLong ${ }^{\text {TM }}$ Gold Antifade Mountant with DAPI (P36935) were acquired from Thermo Fisher Scientific (Waltham, MA, USA). Ki67 (ab15580), IL-6 (ab179570), TNF- $\alpha$ (ab8348), goat anti-mouse IgG H\&L (ab150115) and goat anti-rabbit
IgG H\&L (ab150077) were purchased from Abcam (Cambridge, UK). Paraformaldehyde (4\%) was obtained from Phygene Life Sciences Co., Ltd. (Fuzhou, China). 1, 1'-Dioctadecyl-3,3,3',3'-tetramethylindocarbocyanine (DiI) was from Invitrogen (Carlsbad, CA, USA).

\section{Cell Culture}

An immortalized human skin keratinocyte cell line (HaCaT) was obtained from Dr Hua Yu at the University of Macau, which was purchased from the American Type Culture Collection (ATCC, USA). The cell line was authenticated by Short Tandem Repeat (STR) profiling, which was conducted by Beyotime Biotechnology (Shanghai, China). HaCaT cells were cultured in Dulbecco's modified Eagle's medium with $10 \%(\mathrm{v} / \mathrm{v})$ FBS, $100 \mathrm{U} / \mathrm{mL}$ penicillin, and $100 \mu \mathrm{g} / \mathrm{mL}$ streptomycin. The condition of the incubator is $95 \%$ humidity containing $5 \% \mathrm{CO}_{2}$ at $37^{\circ} \mathrm{C}$.

\section{Animals}

C57/BL6 mice (female, 7-9 weeks) were obtained from the Animal Facility of the University of Macau. The animal protocols were approved by the Animal Ethics Committee of the University of Macau (UMARE-0362018). The experiments followed the NIH Guidelines for the Care and Use of Laboratory Animals.

\section{Preparation and Characterization of Cel Nio}

For the preparation of Cel Nio, the method of thin-film hydration and sonication as described in our previous publication was used. $^{24}$ Briefly, $30 \mathrm{mg}$ of Span 20, $10 \mathrm{mg}$ of Span $60,10 \mathrm{mg}$ of cholesterol, and $2 \mathrm{mg}$ of Cel were dissolved in $4 \mathrm{~mL}$ of chloroform. Then, cholesterol was removed by a rotary evaporator, and the dried lipid layer was hydrated with $5 \mathrm{~mL}$ of Mill-Q water. Subsequently, the suspension was sonicated with an Ultrasonic Cell Crusher (BILON-250Y, Shanghai) at 125 W with a 3-mm probe for $2 \mathrm{~min}$ to acquire Cel Nio.

The particle size distribution, polydispersity index (PDI), and zeta potential of Cel Nio were tested via dynamic light scattering (DLS) (Malvern, UK). The EE\% and drug loading capacity (DL) of Cel Nio were quantified with ultrafiltration. ${ }^{16}$ Briefly, the centrifuge condition of Cel Nio was set at $4000 \times \mathrm{g}$ for $30 \mathrm{~min}$ in a centrifuge tube with a $30-\mathrm{kDa}$ ultrafilter. The total $\mathrm{Cel}$ in the solution of Cel Nio $\left(\mathrm{W}_{\text {total }}\right)$ and the free Cel $\left(\mathrm{W}_{\text {free }}\right)$ were diluted in 
methanol and determined on the HPLC-DAD system (Agilent, USA) with the C18 column (Agilent Zorbax; $4.6 \mathrm{~mm} \times 250 \mathrm{~mm}, 5 \mu \mathrm{m}$ ). The column temperature was $25^{\circ} \mathrm{C}$ and the elution condition was methanol: $0.4 \% \mathrm{H}_{3} \mathrm{PO}_{4}$ solution $(85: 15, \mathrm{v} / \mathrm{v})$. The velocity of flow was $1 \mathrm{~mL} / \mathrm{min}$, and the detection wavelength was $426 \mathrm{~nm}$. Samples injected into the column were $10 \mu \mathrm{L}$. The EE and DL were quantified according to the equation:

$\mathrm{EE}=\left(\mathrm{W}_{\text {total drug }}-\mathrm{W}_{\text {free drug }}\right) / \mathrm{W}_{\text {total drug }} \times 100 \%$ (1)

$\mathrm{DL}=\mathrm{W}_{\text {drug }} / \mathrm{W}_{\text {drug }+ \text { lipid }} \times 100 \%$ (2)

For the investigation of the morphology of Cel Nio, transmission electron microscopy (TEM, Hitachi TEM system, Japan) was used. One drop of diluted Cel Nio suspension was dropped on the copper grid for $1 \mathrm{~min}$, then negative staining was performed with Neutral phosphotungstic acid $(2 \% \mathrm{w} / \mathrm{v})$. The samples were analyzed by TEM with $80 \mathrm{kV}$ accelerating voltage.

\section{Preparation of Cel Nio-Loaded Hydrogel}

Adequate viscosity is necessary for topical formulation to apply on the skin. Carbopol was selected as the gel matrix base of Cel Nio. ${ }^{24}$ Briefly, $1 \%$ (w/v) Carbopol was added to Cel Nio solution and constantly stirred in darkness overnight, then neutralized with triethanolamine. The final concentration of $\mathrm{Cel}$ in $\mathrm{Cel}$ Nio gel was $0.02 \%$. For the preparation of Cel gel and Blank Nio gel, 1\% (w/v) Carbopol was added to Cel or Blank Nio using the same method.

\section{Pharmacokinetic Study of Cel Nio on Mice}

\section{Sample Preparation}

The time points were set according to the previous study on pharmacokinetics and bioactivity of $\mathrm{Cel} .{ }^{25,26} \mathrm{C} 57 \mathrm{BL} / 6$ mice were allocated into five groups (each group for one time point), and Cel Nio gel $(0.4 \mathrm{~g})$ was topically administrated on the shaved skin of mice. Each group has six mice and they were administrated once a day for 6 days. The sixth day before administration was set as the zero point. At each time point $(0,6,12,24,48$ hours postadministration), the blood samples, dorsal skin, draining lymph nodes (DLN), and mesenteric lymph nodes (MLN) were obtained after sacrificed.

After centrifugation of the blood samples at $2000 \times$ $\mathrm{g}$ for $10 \mathrm{~min}$, the supernatant was obtained to be plasma samples. Then, each plasma sample $(100 \mu \mathrm{L})$ was extracted with $200 \mu \mathrm{L}$ of cold ethyl acetate, and the extraction was performed in triplicate. Then the combined supernatants were evaporated by a stream of nitrogen then dissolved with $95 \mu \mathrm{L}$ of acetonitrile aqueous solution, and added $5 \mu \mathrm{L}$ of hydrocortisone (IS, $100 \mathrm{ng} / \mathrm{mL}$ ) solution.

Skin samples were rinsed by phosphate-buffered saline (PBS) and $1 \mathrm{~mL}$ of absolute ethanol was added to $100 \mathrm{mg}$ of skin. For the homogenization of skin samples, a Tissuelyser II Disruption System (Qiagen, USA) was employed at 30 frequency/s for $10 \mathrm{~min}$. After centrifugation at $10,000 \mathrm{rpm}$ at $4^{\circ} \mathrm{C}$ with $5 \mathrm{~min}$, the supernatant was filtered with a $0.22-\mu \mathrm{m}$ filter for experiments. Before determination, $10 \mu \mathrm{L}$ of hydrocortisone (IS, $100 \mathrm{ng} / \mathrm{mL}$ ) solution was added to $190 \mu \mathrm{L}$ of the supernatant of skin samples.

For the preparation of DLN and MLN samples, DLN and MLN were weighed and minced using a Tissuelyser II Disruption System (Qiagen, USA) in $500 \mu \mathrm{L}$ of absolute ethanol at a frequency of 30 times/s for $5 \mathrm{~min}$ and centrifuged at $22,000 \times \mathrm{g}$ with $10 \mathrm{~min}$ to obtain the supernatant. Then dried the supernatant under a stream of nitrogen and dissolved with three volumes of dry ethanol to obtain the DLN and MLN samples. Before determination, $5 \mu \mathrm{L}$ of hydrocortisone (IS, $100 \mathrm{ng} / \mathrm{mL}$ ) solution was added to $95 \mu \mathrm{L}$ of DLN or MLN samples.

\section{LC-MS Analysis}

The separation of samples was conducted with the Agilent 1290 Infinity ultra-performance liquid chromatography system (UHPLC, USA) equipped with an Agilent Eclipse plus-C18 column $(2.1 \times 100 \mathrm{~mm}, 1.8 \mu \mathrm{m})$. The column temperature was $40^{\circ} \mathrm{C}$. The sample injected was $5 \mu \mathrm{L}$. The mobile phases A and B consisted of water and acetonitrile, both of which were added with $0.1 \%$ formic acid, and the linear gradient was as follows: $0-5 \mathrm{~min}, 10-50 \% \mathrm{~B} ; 5-6$ min, 50-85\% B; 6-10 min, 85-95\% B; 10-13 min, 95$95 \%$ B. The velocity of flow was $0.35 \mathrm{~mL} / \mathrm{min}$. The quantitative equipment was the Agilent $6490 \mathrm{iFunnel}$ triple quadrupole mass system (QQQ-MS) equipped with a dual Jet Stream electrospray ion source (dual AJS ESI). The positive ion mode with a monitoring reaction mode (MRM) method was selected to quantify the concentration of Cel. The other MS parameters were set as follows: The gas temperature at $250{ }^{\circ} \mathrm{C}$, gas flow at $15 \mathrm{~L} / \mathrm{min}$, nebulizer pressure at $25 \mathrm{psig}$, sheath gas temperature at $300{ }^{\circ} \mathrm{C}$, sheath gas flow at $12 \mathrm{~L} / \mathrm{min}$, nozzle voltage at $450 \mathrm{~V}$, capillary at $4500 \mathrm{~V}$. The iron transition was $\mathrm{m} / z 451.3 \rightarrow$ 201.1 (24 eV collision energy), and hydrocortisone (IS) was $m / z 363.1 \rightarrow 121.2$ transition ( $20 \mathrm{eV}$ collision energy). 


\section{The Antipsoriatic Effect of Cel Nio Gel in IMQ-Induced Psoriatic Mice \\ Topical Administration in IMQ-Induced Psoriatic \\ Mice}

The IMQ-induced psoriatic mouse model was established with C57BL/6 mice regarding our previous study. ${ }^{25}$ The mice were randomly allocated into six groups. Briefly, except for the normal group, IMQ cream (containing $3.125 \mathrm{mg}$ of the active ingredient) was applied to the shaved dorsal skin of mice in the morning each day for 6 days. The group treated with IMQ only was the negative control group. The preparations were applied to the dorsal skin of mice respectively $6 \mathrm{~h}$ after administration of IMQ cream. The group treated with IMQ and tacrolimus cream was the positive control group. The other four groups were respectively administrated with Blank Nio gel, Cel gel, or Cel Nio gel each day for 6 days. The dosage of Cel was $0.08 \mathrm{mg}$ for each mouse per day, while that of tacrolimus was $0.1 \mathrm{mg}$ for each mouse per day.

\section{Psoriasis Area Severity Index (PASI) Evaluation}

For the evaluation of the severity of skin lesions, the PASI method was adopted. ${ }^{26}$ The severity of erythema and scaling ranged from 0 to $4(0$, none; 2 , moderate; 3 , marked; and 4 , severe). The PASI was the average of two observers' independent scores in a blinded fashion. The total scores were the sum of erythema and scaling scores. PASI evaluations were performed daily for 7 days.

\section{Weight Ratio of the Spleen to the Body (Spleen/Body wt\%)}

The weight of the spleen is considered to be an important indicator of immune activation, because the increase in spleen/body wt $\%$ probably reflects the increase of immune cells in the spleen. ${ }^{27}$ The weights of the mice were weighed in advance of sacrifice, and the spleens were obtained immediately after sacrifice, then the spleen/body $\mathrm{wt} \%$ was calculated.

\section{Evaluation of Serum Cytokine Levels}

Blood samples were obtained from each mouse after sacrifice. After centrifugation at $4000 \mathrm{rpm}$ with $15 \mathrm{~min}$, the supernatant was obtained as the serum samples. The Cytometric Bead Array (CBA) with a BD CBA Mouse Th1/Th2/Th17 Cytokine Kit (BD Bioscience, Catalog No. 560485) was applied for detection of TNF, IL-2, IFN- $\gamma$, IL-6, and IL-17A. Briefly, the same volumes of mixed beads, serum samples, and $\mathrm{PE}$ detection antibodies were mixed and incubated with $2 \mathrm{~h}$ at $25^{\circ} \mathrm{C}$ in darkness.
Subsequently, the samples were rinsed and then analyzed with a BD LSRF Fortessa flow cytometer with FACP Array Software (BD Biosciences, San Jose, CA, USA).

\section{In vitro Uptake by $\mathrm{HaCaT}$ Cells}

The HaCaT cells $\left(2 \times 10^{5}\right.$ cells/well $)$ were seeded in the 12 well plate and cultured overnight. Then, the HaCaT cells were incubated with free DiI $(1 \mu \mathrm{g} / \mathrm{mL})$ and DiI-Nio for 8 h. After that, the cells were digested by trypsin and collected. The DiI fluorescence in the HaCaT cells was quantified with flow cytometry (BD LSR Fortessa, BD, USA).

\section{The Anti-Inflammatory Effect of Cel on $\mathrm{HaCaT}$ Cells}

HaCaT cells were seeded in 12 -well plates $\left(2 \times 10^{5}\right.$ cells/ well), with stimulation of $10 \mu \mathrm{g} / \mathrm{mL}$ IMQ to activate inflammation. The concentration of IMQ was set with the reference to a previous study on the activation of keratinocytes by IMQ. ${ }^{28}$ Meanwhile, $0.25 \mu \mathrm{M}$ Cel was applied to the cells for $6 \mathrm{~h}$. Afterward, total mRNA from HaCaT cells was extracted with Trizol (Invitrogen, USA), then cDNA was obtained with a PrimeScript RT Reagent Kit (TaKaRa, Bio Inc.). For quantification of real-time PCR (qPCR), SYBR Premix Ex Taq II (TaKaRa, Bio Inc.) was used on the Vii 7 qPCR System (Applied Biosystem, USA). The sequences of the primers are listed in Table 1: The expression levels of genes were analyzed using the $2^{-\triangle \triangle \mathrm{CT}}$ method relative to the GAPDH gene.

\section{Immunofluorescence Analysis of Mouse Skin}

The thickness of the dorsal skin of mice was sliced into 10 $\mu \mathrm{m}$, and the sections were fixed in $4 \%$ paraformaldehyde and rinsed with PBS. For the block of nonspecific antibody binding, $10 \%$ goat serum was added then incubated for 1 hour at ambient temperature. Primary antibodies against Ki-67 (ab15580, Abcam, 1:200), IFN- $\beta$ (PA5-20390, Thermo Fisher, 1:100), IL-6 (ab179570, Abcam, 1:50) and TNF- $\alpha$ (ab8348, Abcam, 1:100) were incubated with the samples overnight at $4^{\circ} \mathrm{C}$. The sections were incubated with the secondary antibody (Alexa Fluor ${ }^{\circledR} 488$ or Alexa Fluor $^{\mathbb{B}}{ }^{647)}$ for 1 hour at ambient temperature. Nuclei were stained with ProLong ${ }^{\text {TM }}$ Gold Antifade Mountant with DAPI (P36935, Thermo Fisher). Immunofluorescence was observed with confocal laser microscopy (CLSM) (Leica TCS SP8 multiphoton microscope, USA). 
Table I Primer Sequences of Human Genes Examined by Quantitative Real-Time PCR

\begin{tabular}{|l|l|}
\hline Primer & Base Sequence (5' to $\mathbf{3}^{\prime}$ ) \\
\hline IL-6 (S) & ACTCACCTCTTCAGAACGAATTG \\
IL-6 (AS) & CCATCTTTGGAAGGTTCAGGTTG \\
TNF- $\alpha$ (S) & CCTCTCTCTAATCAGCCCTCTG \\
TNF- $\alpha$ (AS) & GAGGACCTGGGAGTAGATGAG \\
IFN- $\beta$ (S) & GCTTGGATTCCTACAAAGAAGCA \\
IFN- $\beta$ (AS) & ATAGATGGTCAATGCGGCGTC \\
GAPDH (S) & CTGGGCTACACTGAGCACC \\
GAPDH (AS) & AAGTGGTCGTTGAGGGCAATG \\
\hline
\end{tabular}

\section{Statistical Analysis}

Significant differences between different groups were analyzed via one-way ANOVA with repeated measures using GraphPad Prism 7.

\section{Results}

\section{Characterization of Cel Nio}

All the Cel Nios showed a nearly spherical shape with the particle size of $133 \mathrm{~nm} \pm 2.1 \mathrm{~nm}$, and the PDI of $0.249 \pm$ 0.02 (Figure 1A and B). The zeta potential was $-37.5 \mathrm{mV}$ $\pm 1.2 \mathrm{mV}$, and the particle size had no obvious change within 6 months, indicating good nanoparticle stability (Figure 1C). Notably, we further found that Cel Nio displayed EE\% and DL\% of $83.2 \pm 1.15 \%$ and $3.33 \pm$ $0.05 \%$, respectively. After prepared into gel, Cel Nio gel had proper viscosity and could be easily applied to the skin, which could improve the retention effect on the skin. The photograph of Cel Nio gel was shown in Figure 1D.

\section{Pharmacokinetic Study of Cel Nio Gel on Mice}

To explore the target site of Cel Nio gel, the pharmacokinetics were subsequently investigated by detecting the concentrations of $\mathrm{Cel}$ in the skin, plasma, DLN, and MLN at different time points after topical delivery of Cel Nio gel to the skin, using a sensitive LC-MS method. Data showed that Cel was mainly retained in the skin, and only traceable Cel could be detected in blood circulation and the lymphatic system (Table 2), indicating that there was little systemic absorption after topical delivery of Cel Nio gel on the skin.
A

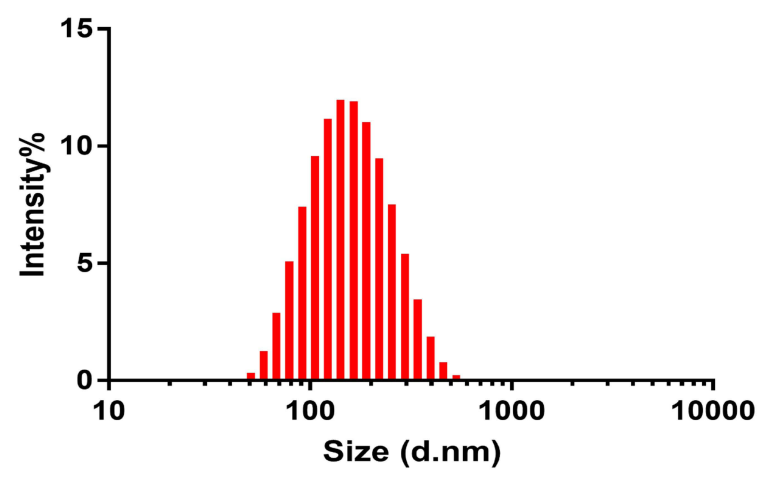

C

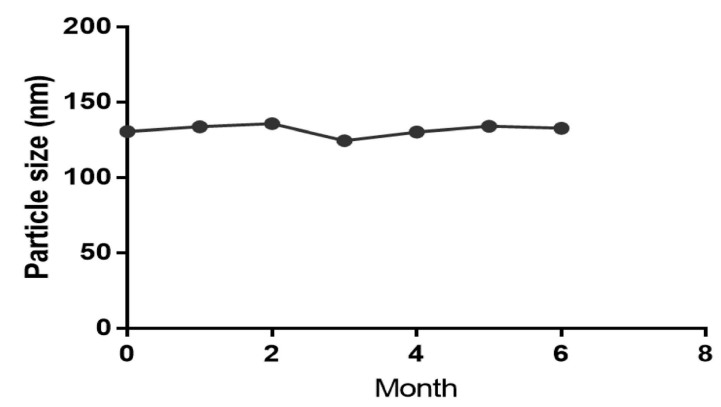

B

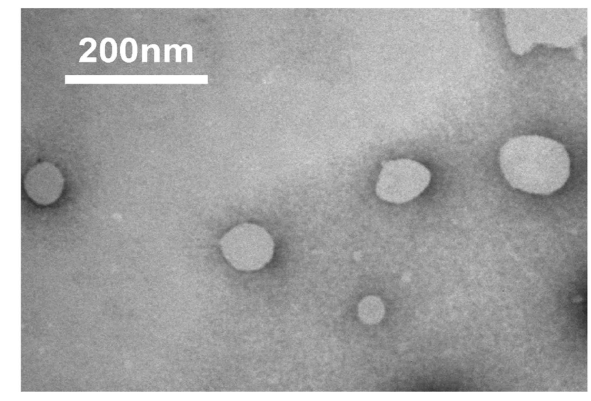

D

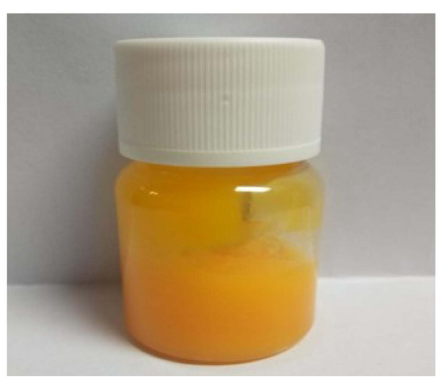

Figure I Characterization of Cel Nio. (A) Particles distribution of Cel Nio; (B) TEM of Cel Nio (The bar is $200 \mathrm{~nm}$ ); (C) The stability of Cel Nio of 6 months; (D) The photograph of Cel Nio gel. 
Table 2 Cel Content in the Skin, Plasma, Draining Lymph Nodes (DLN) and Mesenteric Lymph Nodes (MLN) of Mice After Administration of Cel Nio Gel $(0.4 \mathrm{~g})$

\begin{tabular}{|l|r|r|r|r|}
\hline Time Points & \multicolumn{1}{|c|}{ C Cel in Skin $_{\text {(pg/mg) }}$} & \multicolumn{1}{c|}{ C Cel in Plasma $(\mathbf{p g} / \mathbf{m g})$} & \multicolumn{1}{c|}{ C Cel in DLN $_{\text {(pg/mg) }}$} & C Cel in MLN (pg/mg) \\
\hline $0 \mathrm{~h}$ & $(0.301 \pm 0.055) \times 10^{3}$ & $0.700 \pm 0.232$ & $1.343 \pm 0.546$ & $0.263 \pm 0.429$ \\
$6 \mathrm{~h}$ & $(0.368 \pm 0.086) \times 10^{3}$ & $1.165 \pm 0.196$ & $2.513 \pm 1.189$ & $0.518 \pm 0.443$ \\
$12 \mathrm{~h}$ & $(0.523 \pm 0.056) \times 10^{3}$ & $2.385 \pm 0.655$ & $1.688 \pm 0.359$ & $0.720 \pm 0.482$ \\
$24 \mathrm{~h}$ & $(0.311 \pm 0.123) \times 10^{3}$ & $0.898 \pm 0.340$ & $1.350 \pm 0.843$ & $0.383 \pm 0.320$ \\
$48 \mathrm{~h}$ & $(0.255 \pm 0.136) \times 10^{3}$ & $0.303 \pm 0.080$ & $0.690 \pm 0.350$ & $0.120 \pm 0.122$ \\
\hline
\end{tabular}

Note: Data are shown as mean \pm SE $(n=4)$.

Abbreviations: Cel, celastrol; Nio, niosomes.

\section{Histology Evaluation}

The photographs of skin lesions in different groups are demonstrated in Figure 2. The skin of mice in the IMQ only group displayed apparent inflammation with desquamation and erythema. After administration with Blank Nio gel or Cel gel, the white desquamation and erythema on the inflamed skin were ameliorated to some extent. As discovered previously, we confirmed that Cel Nio gel could obviously reduce the white scales and erythema of skin in IMQ-induced psoriasis mice. More importantly, the psoriatic symptoms in the Cel Nio gel group were much milder than those in the tacrolimus group. In addition, we found that the PASI evaluation (Figure S1 in supplementary materials) and the weight ratio of the spleen to the body (Figure S2 in supplementary materials) after administration of Cel Nio gel were both significantly improved.

\section{Evaluation of Serum Cytokine Levels}

As mentioned above, topical administration is considered a good way to reduce systemic toxicity. To investigate whether Cel Nio gel can achieve the anti-inflammatory activity systemically after topical administration, we evaluated the effect of Cel Nio gel on the expression of key inflammatory cytokines (IL-6, IL-17A, TNF- $\alpha$, IL-2, and IFN- $\gamma$ ) in the serum of IMQ-induced psoriatic mice. As summarized in Figure 3, the levels of these cytokines were all obviously higher in the IMQ only group compared to the normal group. Cel gel only showed a significant decrease in the expression level of TNF- $\alpha$, with little effect on IFN- $\gamma$ expression. In sharp contrast, Cel Nio gel could obviously decrease the expression level of IFN- $\gamma$ as well as the other cytokines in the serum of psoriatic mice. Besides, for the secretion of IL- 6 and TNF- $\alpha$, the Cel

\section{Timeline}

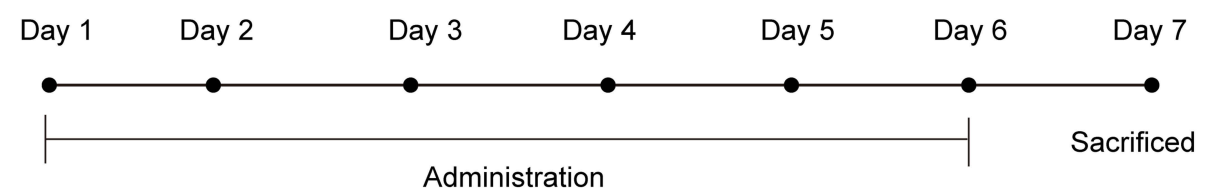

Normal

IMQ treatment

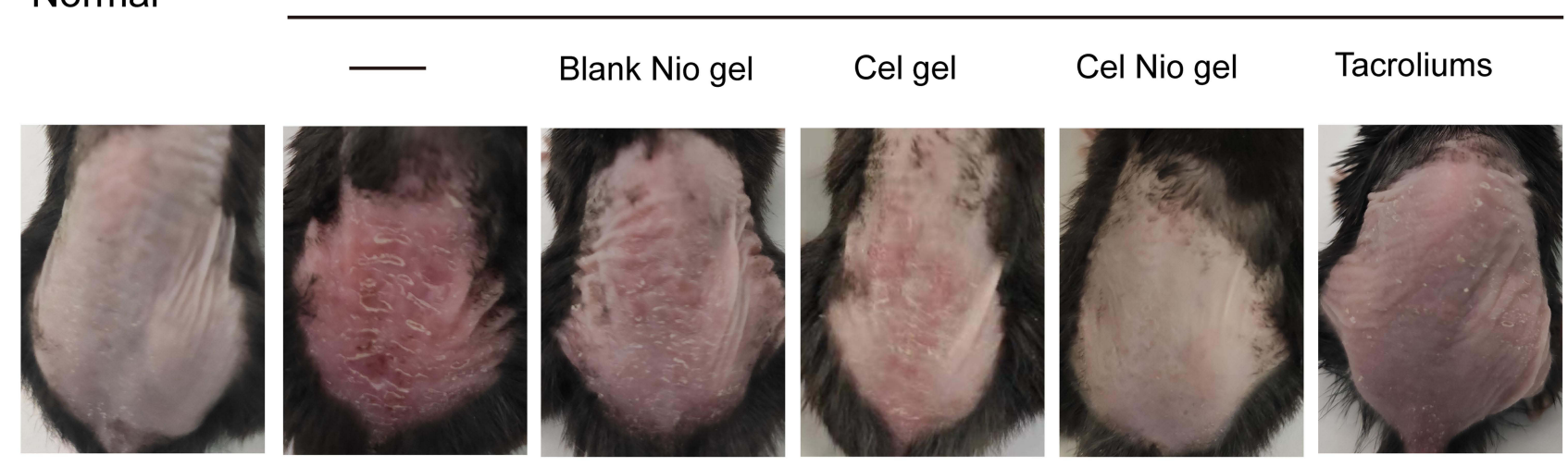

Figure 2 Psoriatic symptoms on the dorsal skin of mice after IMQ and different formulations $(n=6)$. Abbreviations: Cel, celastrol; Nio, niosomes. 

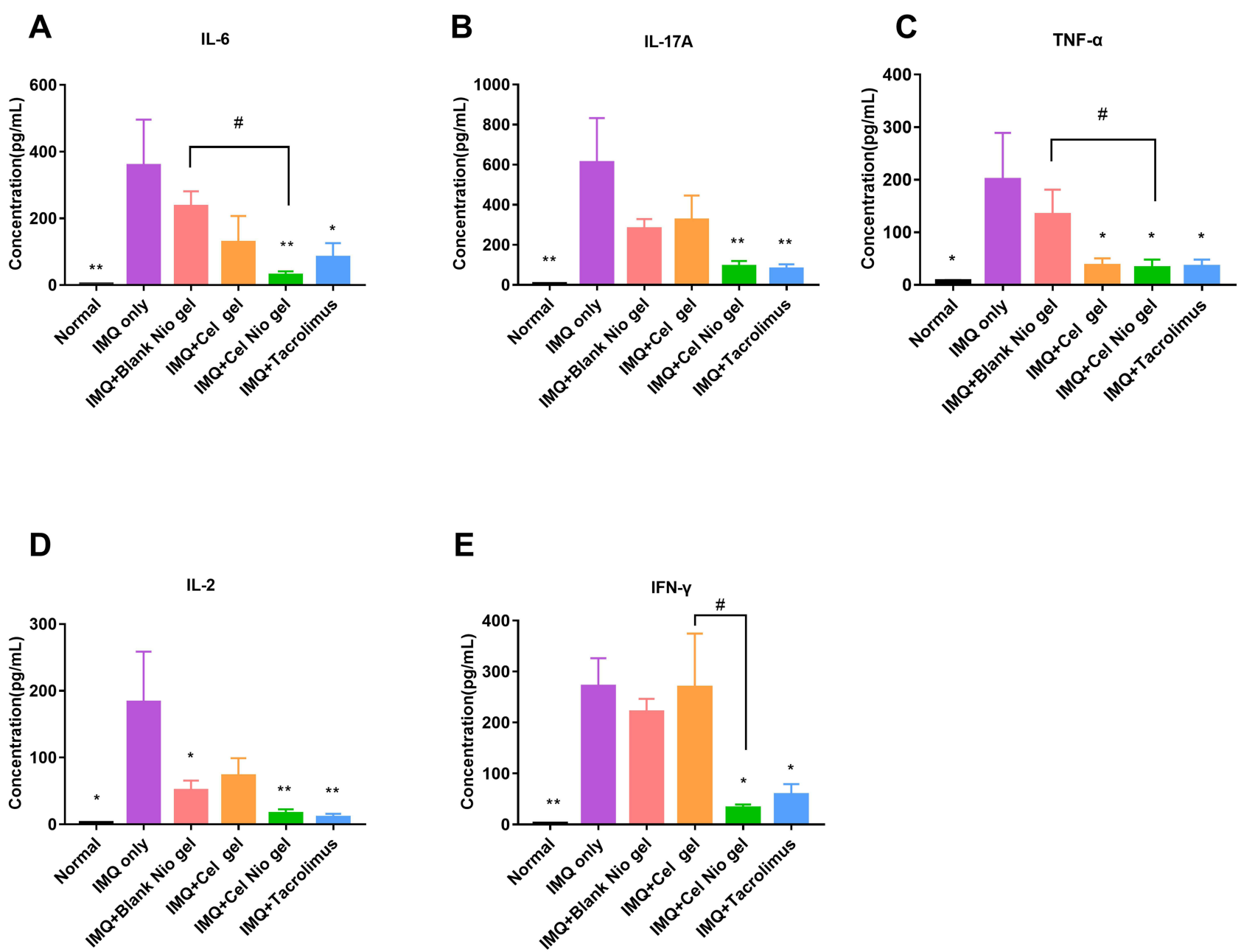

Figure 3 Inflammatory cytokine levels in the serum of mice after treatment of IMQ and different formulations. (A) IL-6; (B) IL-I7A; (C) TNF- $\alpha$; (D) IL-2; (E) IFN- $\gamma$. $*_{p}<0.05 * * p<0.01$, compared with IMQ only group; ${ }^{*}<<0.05$, compared with Cel Nio group. Values are shown as mean and SE ( $\mathrm{n}=6$ ).

Abbreviations: Cel, celastrol; Nio, niosomes.

Nio gel group suggested a significant anti-inflammatory effect compared with the Blank Nio gel group.

\section{In vitro Uptake by HaCaT Cells}

Keratinocytes are crucial in the pathogenesis of psoriasis. $\mathrm{HaCaT}$ cells were human keratinocytes and used as the in vitro model. The amounts of DiI uptaken by HaCaT cells were illustrated in Figure 4. The result showed that the cellular uptake of the DiI-Nio was much higher than that of the free DiI, indicating that the preparation could be effectively uptaken by the $\mathrm{HaCaT}$ cells, subsequently take effects on the HaCaT cells.

\section{The Anti-Inflammatory Effect of Cel on $\mathrm{HaCaT}$ Cells}

To explore the mechanism of the anti-psoriasis activity of $\mathrm{Cel}$, we measured the mRNA levels of inflammatory cytokine genes (IL-6, TNF- $\alpha$, and IFN- $\beta$ ) in HaCaT cells by qRT-PCR. As implied in Figure 5, IMQ significantly increased the mRNA levels of these cytokines in HaCaT cells, suggesting that the inflammation in HaCaT cells was stimulated by IMQ. Interestingly, the mRNA levels of these cytokines were obviously decreased after treatment with Cel for $6 \mathrm{~h}$. The results indicated that Cel could inhibit the inflammation of $\mathrm{HaCaT}$ cells induced by IMQ.

\section{Immunofluorescence Analysis of Mouse}

Skin

To further investigate the anti-inflammation and antihyperproliferation effect of Cel Nio gel on mice, an immunofluorescence analysis was conducted. Immunofluorescence images of Ki-67, IL-6, IFN- $\beta$, and TNF- $\alpha$ in the skin of psoriatic mice are shown in Figure 6. The epidermis of the IMQ-induced area was much thicker than that in the normal 
A

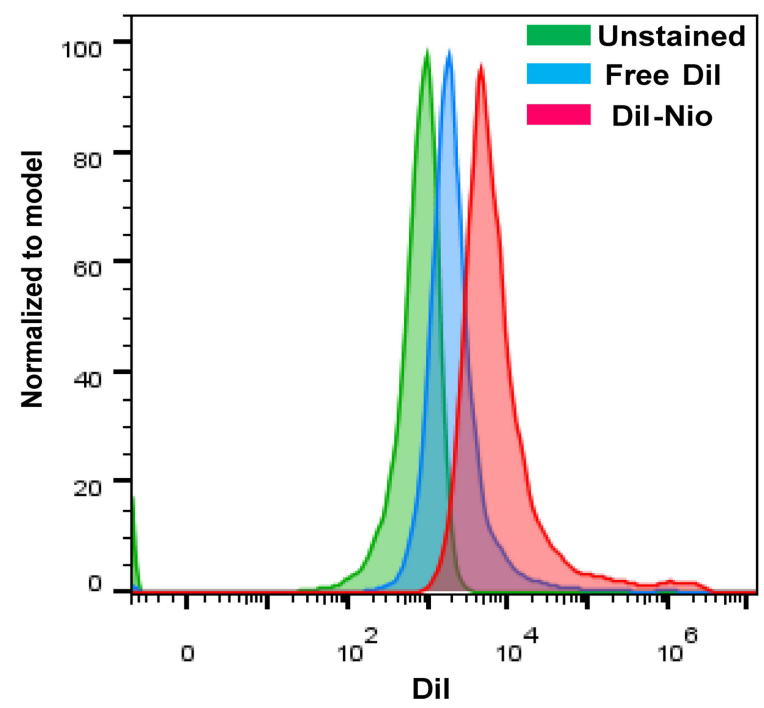

B

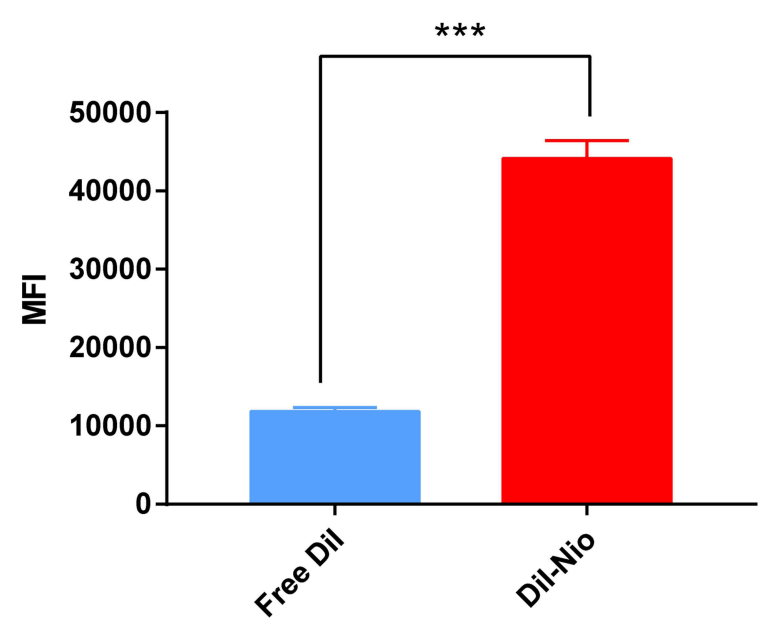

Figure 4 The uptake of free Dil and Dil-Nio by the HaCaT cells. (A) The histogram profiles. (B) Mean fluorescence intensity of the HaCaT cells treated with free Dil and Dil-Nio. ${ }^{* * *} p<0.001$, compared with Free Dil group. Values are shown as mean and SD $(n=3)$.

Abbreviation: Nio, niosomes.
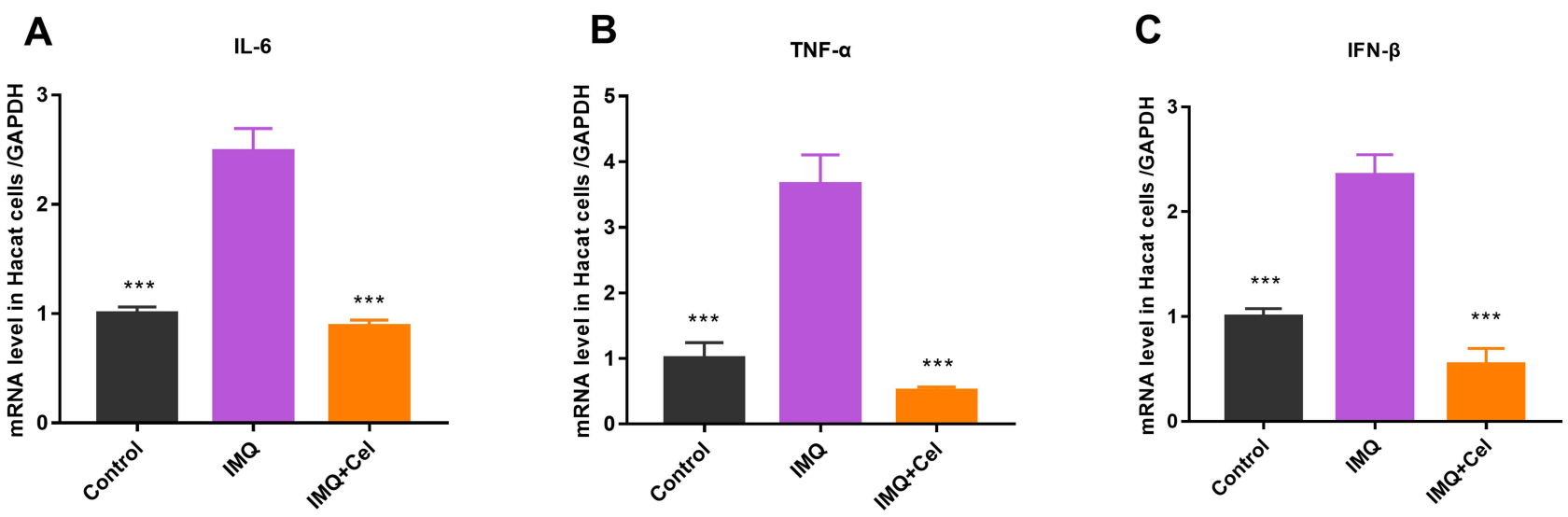

Figure 5 The mRNA levels of cytokines in the HaCaT cell after stimulation of IMQ and treatment with or without Cel. (A) IL-6; (B) TNF- $\alpha$; (C) IFN- $\beta$. *** $p<0.00$ I, compared with IMQ group. Values are shown as mean and SE $(n=6)$.

Abbreviations: Cel, celastrol; Nio, niosomes.

group, which was due to the abnormal proliferation of keratinocytes. This symptom was significantly alleviated by the administration of Cel Nio gel. In addition, Ki-67 in the IMQ only group had higher expression compared with the normal group, indicating excessive proliferation of keratinocytes in the skin lesions. Data showed that Blank Nio gel and Cel gel could only slightly decrease the expression of $\mathrm{Ki}-67$, while the expression of Ki-67 had a significant decline in the Cel Nio gel group.

In addition, the immunofluorescence staining results showed that IMQ increased the protein expression of TNF- $\alpha$, IL-6, and IFN- $\beta$ in the mice. The expression of these cytokines declined slightly in the Blank Nio gel group. There was also a small reduction in the expression of the cytokines with administration of Cel gel. In contrast, there was a significant decline in the expression of the cytokines after therapy of Cel Nio gel. Among them, the expression of TNF- $\alpha$ in vivo was not significantly changed in the epidermis, but mainly in the dermis.

\section{Discussion}

Nano formulations were reported to improve the solubility and skin penetrability of drug in the treatment of psoriasis. ${ }^{29,30}$ Nio provides a vesicle delivery system for higher retention of drugs on the skin compared with conventional dosage forms. ${ }^{31}$ Cel Nio gel was developed for 


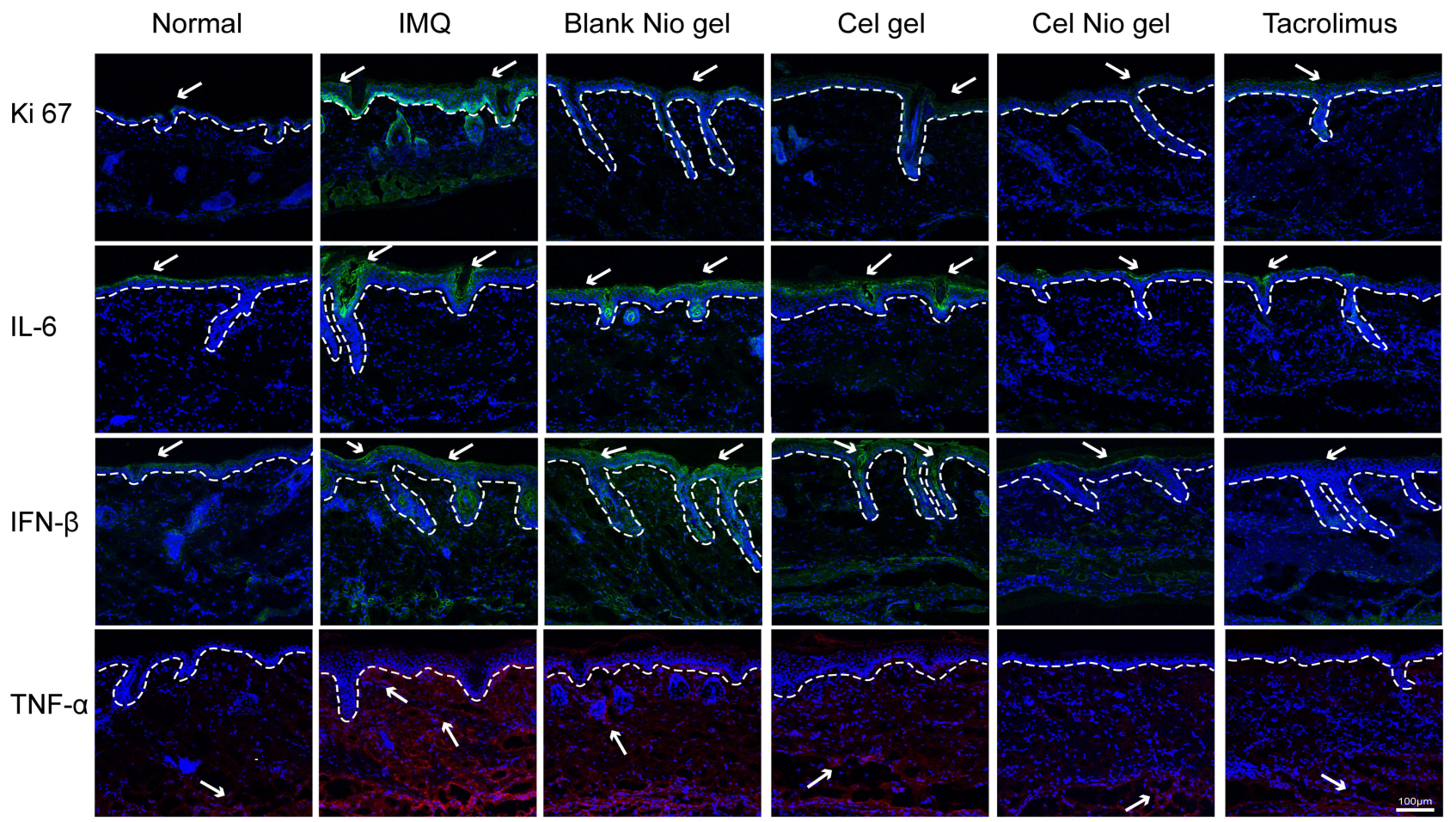

Figure 6 Expression of Ki-67, IL-6, IFN- $\beta$, and TNF- $\alpha$ of skin lesions after the treatment of IMQ and other formulations ( $n=6$ ). Blue, DAPI; Green, Alexa Fluor 488; Red, Alexa Fluor 647. The dotted line demonstrates the border between the epidermis and dermis. Arrows label the representative positive location. Scale bar: $100 \mu \mathrm{m}$. Abbreviations: Cel, celastrol; Nio, niosomes.

the treatment of IMQ-induced psoriasis in our previous work; however, whether Cel Nio gel exerts antipsoriatic activity by topical or systemic effects was not clarified, the mechanism of the anti-psoriasis effect also remained questions.

In this study, we prepared Cel Nio gel and studied its therapeutic target as well as anti-psoriasis mechanism. Since psoriasis is an immune-mediated disease that has a close association with the immune responses of the lymphatic system, ${ }^{32}$ we quantified the concentration of $\mathrm{Cel}$ in the lymphatic system, skin, and blood circulation. The results of pharmacokinetic demonstrated that the encapsulation of Cel into Nio can provide effective concentrations in the skin rather than being absorbed into the blood or lymphatic system. Hence, the encapsulation of $\mathrm{Cel}$ into Nio resulted in low drug concentrations in the blood and lymph system, which minimized potential Cel-related systemic toxicity.

The topical and systemic antipsoriatic effect of Cel Nio gel in the IMQ-induced psoriasis mice was also studied. Cel Nio gel markedly ameliorated the erythema and scales induced by IMQ in the dorsal skin. Meanwhile, the PASI scores and spleen/body wt $\%$ were also reduced after treatment with Cel Nio gel. The levels of inflammatory cytokines in skin lesions were obviously decreased after administration of Cel Nio gel. ${ }^{24}$ Interestingly, in this study, we further found that the levels of inflammatory cytokines in serum were also decreased significantly due to the application of Cel Nio gel. In addition, compared to Blank Nio gel and Cel gel group, Cel Nio gel group suggested a more significant effect on the decrease in inflammatory cytokines level, demonstrated that the preparation of Cel Nio has a better anti-inflammatory effect than the other two preparations. Taken together, these findings revealed that $\mathrm{Cel}$ Nio gel can improve the topical and systemic inflammation of psoriasis via topical delivery without systemic drug exposure, which will enhance the drug therapeutic index and reduce potential systemic toxicity. Besides, the anti-psoriasis effect of Cel Nio by systemic administration was not conducted in this study, which may further demonstrate the advantages of topical delivery.

Due to the pivotal functions of keratinocytes in the pathogenesis of psoriasis, regulation of the inflammatory events induced by keratinocytes could be a relevant strategy to cure psoriasis. ${ }^{33}$ Hence, we further studied the uptake of Nio and anti-inflammatory effects of $\mathrm{Cel}$ on the keratinocytes. Data revealed that the preparation of Nio can enhance the uptake by $\mathrm{HaCaT}$ cells in vitro, which contributes to exerting effects on $\mathrm{HaCaT}$ cells. Furthermore, the study suggested that the expression of inflammatory factors (TNF- $\alpha$, IL- 6 , and IFN- $\beta$ ) 
in HaCaT cells were significantly decreased after the administration of Cel compared to the IMQ only group. The results demonstrated that $\mathrm{Cel}$ inhibited the inflammatory response of keratinocytes in vitro. In addition, the immunofluorescence result revealed that Cel Nio decreased the expression of IL-6 and IFN- $\beta$ in the epidermis of psoriasis mice. Whereas the expression of TNF- $\alpha$ mainly showed in the dermis under psoriasis condition and was inhibited by Cel Nio gel. This phenomenon may be due to that Cel initially inhibited the inflammation of keratinocytes and then reduced the TNF- $\alpha$ secreted by the downstream immune cells in the dermis. Taken together, these findings implied that Cel Nio can improve psoriasis based on inhibiting the inflammation of keratinocytes. Hence, Cel Nio may exert the topical and systemic anti-psoriatic effect by inhibiting the inflammation of keratinocytes in the skin, then further inhibiting the inflammation of DC and T cells in the immune system. ${ }^{5}$

In addition, the expression of $\mathrm{Ki}-67$ has been a marker for evaluating keratinocyte hyperproliferation in psoriasis treatment. ${ }^{34}$ Hence, we analyzed the expression of Ki-67 in IMQ-induced psoriatic mice by immunofluorescence. By comparison to the IMQ-only group, the Cel Nio gel group demonstrated an obvious reduction in $\mathrm{Ki}-67$ expression, indicating that it could also ameliorate psoriatic symptoms by inhibiting the hyperproliferation of keratinocytes in skin lesions.

\section{Conclusions}

The pharmacokinetic study demonstrated that Cel accumulated mainly in the skin without much drug exposure in the blood and lymphatic system. Meanwhile, Cel Nio gel improved the histology evaluation, PASI scores, and spleen/body $\mathrm{wt} \%$ of IMQ-induced psoriatic mice. In addition, Cel Nio gel obviously declined the expression levels of inflammatory cytokines both in skin and blood circulation. These results indicated that although Cel was barely absorbed into the blood or lymphatic system, Cel Nio gel could exert excellent systemic antipsoriasis effects. Moreover, we found that Cel Nio gel achieved the antipsoriatic effect by inhibiting the inflammation and hyperproliferation of keratinocytes in skin lesions and may further suppress systemic inflammation, which may be due to the interactions between keratinocytes and downstream DC and $\mathrm{T}$ cells in the immune system. Cel Nio gel exerted antipsoriatic effects both locally and systemically without obvious systemic exposure, which may provide an example for the design of topical drug delivery systems to avoid systemic toxicity in the psoriasis treatment.

\section{Acknowledgments}

This study was supported by Research Grants from the Macau Science and Technology Development Fund (0013/ 2018/A1), the Research Grant of the University of Macau (MYRG2019-00032-ICMS), and the 2020 Guangdong Provincial Science and Technology Innovation Strategy Special Fund (Guangdong-Hong Kong-Macau Joint Lab), No. 2020B1212030006. We thank the members of the FHS Animal Facility at the University of Macau for the experimental and technical support. We appreciate Meng $\mathrm{Xu}$ for the help of the drawing graphical abstract.

\section{Disclosure}

The authors report no conflicts of interest in this work.

\section{References}

1. Michalek IM, Loring B, John SM, Global report on psoriasis. World Health Organization; 2016.

2. Deng Y, Chang C, Lu Q. The inflammatory response in psoriasis: a comprehensive review. Clin Rev Allergy Immunol. 2016;50 (3):377-389. doi:10.1007/s12016-016-8535-x

3. Greb JE, Goldminz AM, Elder JT, et al. Psoriasis. Nat Rev Dis Primers. 2016;2(1):16082. doi:10.1038/nrdp.2016.82

4. Amani H, Shahbazi MA, D'Amico C, et al. Microneedles for painless transdermal immunotherapeutic applications. J Control Release. 2021;330:185-217. doi:10.1016/j.jconrel.2020.12.019

5. Albanesi C, Madonna S, Gisondi P, et al. The interplay between keratinocytes and immune cells in the pathogenesis of psoriasis. Front Immunol. 2018;9:1549. doi:10.3389/fimmu.2018.01549

6. Zhang LJ, Sen GL, Ward NL, et al. Antimicrobial peptide LL37 and MAVS signaling drive interferon-beta production by epidermal keratinocytes during skin injury. Immunity. 2016;45(1):119-130. doi:10.1016/j.immuni.2016.06.021

7. Wang A, Bai Y. Dendritic cells: the driver of psoriasis. J Dermatol. 2020;47(2):104-113. doi:10.1111/1346-8138.15184

8. Boehncke W-H, Schön MP. Psoriasis. Lancet. 2015;386 (9997):983-994. doi:10.1016/S0140-6736(14)61909-7

9. Hoffman MB, Hill D, Feldman SR. Current challenges and emerging drug delivery strategies for the treatment of psoriasis. Expert Opin Drug Deliv. 2016;13(10):1461-1473. doi:10.1080/17425247.2016.1188801

10. Kim J, Krueger JG. Highly effective new treatments for psoriasis target the IL-23/Type $17 \mathrm{~T}$ cell autoimmune axis. Annu Rev Med. 2017;68:255-269. doi:10.1146/annurev-med-042915-103905

11. Wu JJ, Lynde CW, Kleyn CE, et al. Identification of key research needs for topical therapy treatment of psoriasis - a consensus paper by the international psoriasis council. J Eur Acad Dermatol Venereol. 2016;30(7):1115-1119. doi:10.1111/jdv.13614

12. Menter A, Korman NJ, Elmets CA, et al. Guidelines of care for the management of psoriasis and psoriatic arthritis. Section 3. Guidelines of care for the management and treatment of psoriasis with topical therapies. J Am Acad Dermatol. 2009;60(4):643-659. doi:10.1016/j. jaad.2008.12.032

13. Gu Y, Tang X, Yang M, et al. Transdermal drug delivery of triptolide-loaded nanostructured lipid carriers: preparation, pharmacokinetic, and evaluation for rheumatoid arthritis. Int $J$ Pharm. 2019;554:235-244. doi:10.1016/j.ijpharm.2018.11.024

14. Tao XL, Lipsky PE. The Chinese anti-inflammatory and immunosuppressive herbal remedy Tripterygium wilfordii Hook F. Rheumatic Dis Clin North America. 2000;26(1):29. doi:10.1016/S0889-857X (05)70118-6 
15. Wu C, Jin HZ, Shu D, et al. Efficacy and safety of Tripterygium wilfordii hook $\mathrm{F}$ versus acitretin in moderate to severe psoriasis vulgaris: a randomized clinical trial. Chin Med J (Engl). 2015;128 (4):443-449. doi:10.4103/0366-6999.151069

16. Kang Q, Liu J, Zhao Y, et al. Transdermal delivery system of nanostructured lipid carriers loaded with Celastrol and Indomethacin: optimization, characterization and efficacy evaluation for rheumatoid arthritis. Artif Cells Nanomed Biotechnol. 2018;46 (sup3):S585-S597. doi:10.1080/21691401.2018.1503599

17. Kannaiyan R, Shanmugam MK, Sethi G. Molecular targets of celastrol derived from Thunder of God Vine: potential role in the treatment of inflammatory disorders and cancer. Cancer Lett. 2011;303 (1):9-20. doi:10.1016/j.canlet.2010.10.025

18. Astry B, Venkatesha SH, Laurence A, et al. Celastrol, a Chinese herbal compound, controls autoimmune inflammation by altering the balance of pathogenic and regulatory T cells in the target organ. Clin Immunol. 2015;157(2):228-238. doi:10.1016/j.clim.2015.01.011

19. Zhu H, Ding WJ, Wu R, et al. Synergistic anti-cancer activity by the combination of TRAIL/APO-2L and celastrol. Cancer Invest. 2010;28(1):23-32. doi:10.3109/07357900903095664

20. Freag MS, Saleh WM, Abdallah OY. Self-assembled phospholipid-based phytosomal nanocarriers as promising platforms for improving oral bioavailability of the anticancer celastrol. Int $J$ Pharm. 2018;535(1-2):18-26. doi:10.1016/j.ijpharm.20 17.10 .053

21. Mahale NB, Thakkar PD, Mali RG, et al. Niosomes: novel sustained release nonionic stable vesicular systems-an overview. Adv Colloid Interface Sci. 2012;183-184:46-54. doi:10.1016/j.cis.2012.08.002

22. Kumar GP, Rajeshwarrao P. Nonionic surfactant vesicular systems for effective drug delivery - an overview. Acta Pharmaceutica Sinica B. 2011;1(4):208-219. doi:10.1016/j.apsb.2011.09.002

23. Abdelbary AA, AbouGhaly MH. Design and optimization of topical methotrexate loaded niosomes for enhanced management of psoriasis: application of Box-Behnken design, in-vitro evaluation and in-vivo skin deposition study. Int J Pharm. 2015;485(1-2):235-243. doi:10.1016/j.ijpharm.2015.03.020

24. Meng S, Sun L, Wang L, et al. Loading of water-insoluble celastrol into niosome hydrogels for improved topical permeation and anti-psoriasis activity. Colloids Surf $B$ Biointerfaces. 2019;182:110352. doi:10.1016/j.colsurfb.2019.110352
25. Sun L, Liu Z, Wang L, et al. Enhanced topical penetration, system exposure and anti-psoriasis activity of two particle-sized, curcumin-loaded PLGA nanoparticles in hydrogel. $J$ Control Release. 2017;254:44-54. doi:10.1016/j.jconrel.2017.03.385

26. van der Fits L, Mourits S, Voerman JS, et al. Imiquimod-induced psoriasis-like skin inflammation in mice is mediated via the IL-23/IL17 axis. J Immunol. 2009;182(9):5836-5845. doi:10.4049/jimmun ol.0802999

27. Cesta MF. Normal structure, function, and histology of the spleen. Toxicol Pathol. 2006;34(5):455-465.

28. Weng J-R, Huang T-H, Lin Z-C, et al. Cutaneous delivery of [1-(4-chloro-3-nitrobenzenesulfonyl)-1H-indol-3-yl]-methanol, an indole-3-carbinol derivative, mitigates psoriasiform lesion by blocking MAPK/NF- $\mathrm{kB} / \mathrm{AP}-1$ activation. Biomed Pharmacother. 2019;119:109398. doi:10.1016/j.biopha.2019.109398

29. Algahtani MS, Ahmad MZ, Ahmad J. Nanoemulsion loaded polymeric hydrogel for topical delivery of curcumin in psoriasis. $J$ Drug Deliv Sci Technol. 2020;59:101847. doi:10.1016/j.jddst.2020.101847

30. Algahtani MS, Ahmad MZ, Ahmad J. Nanoemulgel for improved topical delivery of retinyl palmitate: formulation design and stability evaluation. Nanomaterials (Basel). 2020;10(5):848. doi:10.3390/ nano10050848

31. Jacob S, Nair AB, Al-Dhubiab BE. Preparation and evaluation of niosome gel containing acyclovir for enhanced dermal deposition. J Liposome Res. 2017;27(4):283-292. doi:10.1080/08982104.20 16.1224897

32. Chang JE, Turley SJ. Stromal infrastructure of the lymph node and coordination of immunity. Trends Immunol. 2015;36(1):30-39. doi:10.1016/j.it.2014.11.003

33. Albanesi C, De Pita O, Girolomoni G. Resident skin cells in psoriasis: a special look at the pathogenetic functions of keratinocytes. Clin Dermatol. 2007;25(6):581-588. doi:10.1016/j.clindermatol.20 07.08.013

34. Boakye CHA, Patel K, Doddapaneni R, et al. Novel amphiphilic lipid augments the co-delivery of erlotinib and IL36 siRNA into the skin for psoriasis treatment. $J$ Control Release. 2017;246:120-132. doi:10.1016/j.jconrel.2016.05.017
International Journal of Nanomedicine

\section{Publish your work in this journal}

The International Journal of Nanomedicine is an international, peerreviewed journal focusing on the application of nanotechnology in diagnostics, therapeutics, and drug delivery systems throughout the biomedical field. This journal is indexed on PubMed Central, MedLine, CAS, SciSearch ${ }^{\mathbb{R}}$, Current Contents ${ }^{\mathbb{R}} /$ Clinical Medicine, $^{2}$

\section{Dovepress}

Journal Citation Reports/Science Edition, EMBase, Scopus and the Elsevier Bibliographic databases. The manuscript management system is completely online and includes a very quick and fair peer-review system, which is all easy to use. Visit http://www.dovepress.com/ testimonials.php to read real quotes from published authors. 